Introduction Cholangioscopy with electrohydraulic lithotripsy (EHL) is an evolving service to treat choledocholithiasis refractory to standard ERCP and is included in the BSG guidelines. We aimed to assess the safety, efficacy and implications of a new tertiary Spyglass EHL service for treatment of refractory gallstone disease.

Methods A review of prospectively collected data was performed for all EHL Spyglass procedures performed from 01.12.2018 to 31.12.2019 at St Thomas' Hospital. Procedural duration was determined by the time between first and last fluoroscopic image taken. Stone burden was assessed by examination of fluoroscopic images. ERCP Spyglass DS1 and Autolith Touch II EHL Generator was used for all procedures. Results 57 Spyglass EHL procedures were performed between January 2018 and December 2019 on 40 patients $(75 \%$ females, $25 \%$ males with a mean age of 65). All cases had failed stone clearance at standard ERCP prior to this. Mean diameter of the largest stone was $1.83 \mathrm{~cm}$ with a range of 1 $3.5 \mathrm{~cm}$. All stones were $<2 \mathrm{~cm}$ in patients who achieved ductal clearance in 1 procedure.

Out of 40 patients, 9 were excluded as they were awaiting further EHL for duct clearance. 31/31 (100\%) achieved ductal clearance at the last procedure. Ductal clearance was achieved with 1 EHL session in 11/31(35.5\%), 2 EHL sessions in 11/ $31(35.5 \%), 3$ EHL sessions in $7 / 31(23 \%)$ and 4 sessions in 2/ $31(6 \%)$. There was no correlation between number of stones and number or duration of EHL sessions.

The average number of shocks used in one EHL was 1600 shocks. Average time spent on one EHL was 78 minutes. In 2018, 37.1 hours were used for Spyglass EHL in total (this does not include diagnostic Spyglass procedures).

Documented complications occurred in 2/57(3.5\%) procedures; 1 peri-pancreatic abscess with necrotic nodes and 1 lingual haematoma secondary to intubation. There were no reported cases of pancreatitis or 30 day mortality.

Conclusions Cholangioscopy with EHL is an effective treatment for refractory choledocolithiasis, achieving 100\% ductal clearance, with a low rate of complications. However the procedural duration and number of repeat procedures required based on stones size/burden is difficult to predict. This data will be useful to centres planning to roll out a Spyglass-EHL service.

\section{PTU-6 COMPLETION COLONOSCOPY IN PATIENTS WITH ADENOMAS IDENTIFIED ON FLEXIBLE SIGMOIDOSCOPY; IS IT ALWAYS NECESSARY?}

Larissa Good*, Zameer Mohamed, Georgina Chadwick, Carole Collins, Emma Johnston, Samuel Pannick, Moe Kyaw, Krishna Sundaram, Richard Appleby. West Middlesex University Hospital, London, UK

\subsection{6/gutjnl-2021-BSG.79}

Introduction The BSG guidelines on adenoma surveillance make no recommendation for adenomas identified on flexible sigmoidoscopy (FS). Conventionally, a completion colonoscopy is performed for any patient with an adenoma found on FS. However, Bagshaw and colleagues [1] proposed that this could be limited to 'high risk' lesions found at FS. We assessed this risk stratified approach using data from individuals undergoing initial FS and completion colonoscopy for bright per rectal (PR) bleeding.

Methods We retrospectively identified all patients who had an adenoma detected at FS for bright PR bleeding at West
Abstract PTU-6 Table 1 FS and completion colonoscopy outcomes stratified by age and risk findings $(n=29)$

\begin{tabular}{lll}
\hline FS & \multicolumn{2}{l}{ Completion Colonoscopy } \\
\cline { 2 - 3 } & Normal/Low Risk & High Risk/Cancer \\
\hline$<60$ Low Risk Finding & 13 & 0 \\
$<60$ High Risk Finding/Cancer & 5 & 1 \\
$>60$ Low Risk Finding & 6 & 4 \\
$>60$ High Risk/Cancer & 0 & 0 \\
\hline
\end{tabular}

Middlesex University Hospital between May 2019 and January 2020, using the audit function on the local endoscopy software.

In addition to demographic information, the study identified the proportion undergoing completion colonoscopy and polyp characteristics. Polyps were stratified 'high risk' according to features proposed by Bagshaw; tubulovillous adenoma (TVA)/villous adenoma (VA)/sessile serrated lesion (SSL) of any size, high grade dysplasia of any size, $>3$ tubular adenomas (TA), > 20 hyperplastic polyps and persistent rectal bleeding.

Results 74 FS were performed for PR bleeding, with 37 (50\%) having at least 1 adenoma detected. According to the Bagshaw criteria, 4 patients (11\%) had high risk adenoma findings and $1(1 \%)$ had an adenocarcinoma on FS, all were aged <60. $29(78 \%)$ patients with an adenoma at FS had a completion colonoscopy. $5(17 \%)$ had high risk findings or cancer at colonoscopy, of whom only one was $<60$; this patient had a high risk lesion at initial FS. Of the 13 patients $<60$ with low risk findings on FS as per the Bagshaw criteria, 0 patients had high risk findings on completion colonoscopy.

Conclusions No patient under 60 with low risk polys detected at FS, had a subsequent high risk lesion identified on completion colonoscopy. Therefore, the introduction of a risk-based adenoma stratification guidance for completion colonoscopy may prove to be useful among individuals below the National Bowel Cancer Screening Programme age reporting fresh PR bleeding, particularly given ever increasing pressures faced by providers to conduct timely endoscopy procedures.

\section{REFERENCE}

1. Bagshaw $P$, et al. Guidelines for completion colonoscopy after polyps are found at flexible sigmoidoscopy for investigation of haemorrhoidal-type rectal bleeding. Gut 2020.

\section{PTU-7 PREDICTORS OF HIGH-RISK ENDOSCOPIC FEATURES IN PATIENTS WITH ACUTE UPPER GASTROINTESTINAL BLEEDING}

${ }^{1}$ Stephanie Poo*, ${ }^{2}$ Annelies Sweeney, ${ }^{2}$ Kar Mun Ang, ${ }^{2}$ Ahmed El-masry, ${ }^{2}$ Rigers Cama, ${ }^{2}$ Rajan Patel, ${ }^{2}$ Kalpesh Besherdas. 'London North West Healthcare NHS Trust, London, UK; ${ }^{2}$ Royal Free London NHS Trust, London, UK

\subsection{6/gutjnl-2021-BSG.80}

Introduction The Glasgow Blatchford Score is useful in predicting the need for hospital-based intervention following an acute upper GI bleed (AUGIB); however the accuracy of risk scores for determining risk of rebleed or further intervention is relatively low(1). It is well established that high-risk features during endoscopy (HRE) in AUGIB are associated with poor outcomes. We aimed to evaluate pre-endoscopic parameters to 


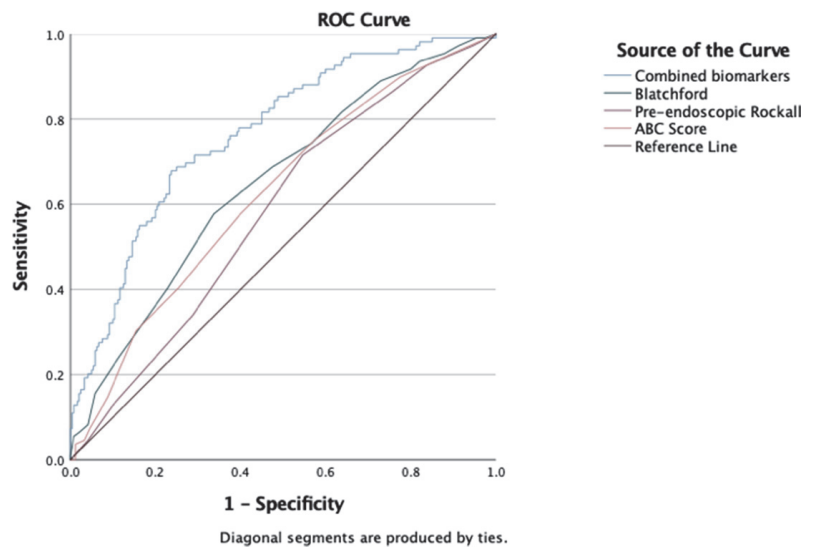

Abstract PTU-7 Figure 1 ROC curve comparing pre-endoscopic scoring systems in predicting HRE

predict patients with high-risk features during endoscopy and therefore poorer prognosis.

Methods We conducted a retrospective analysis of patients presenting with emergency AUGIB at Barnet Hospital over 22 months, using electronic patient records and endoscopy database. HRE were defined as: Forrest $1 \mathrm{~A} / 1 \mathrm{~B} / 2 \mathrm{~A} / 2 \mathrm{~B}$ ulcers, Grade 2/3 varices, malignant lesion, and need for endotherapy. Statistical analyses conducted include Chi-Square tests for associations, multi-variable analysis and ROC curve; a p-value of $<0.05$ was considered statistically significant.

Results 153 of 473 (32\%) patients had HRE (66\% male, median age 73 years). Significant presenting complaints included melaena in $62.9 \% \quad(\mathrm{p}=0.001)$, haematemesis in $31.7 \%(\mathrm{p}=0.001)$, syncope in $20.5 \%(\mathrm{p}=0.012)$ and exertional dyspnoea in $13.2 \% \quad(\mathrm{p}=0.000)$. A history of cirrhosis and prior malignancy were significantly associated with HRE $(\mathrm{X} 2=5.516, \mathrm{p}=0.019$ and $\mathrm{X} 2=5.186, \mathrm{p}=0.023$ respectively). Multi-regression analyses showed that variables predicting HRE include haematemesis (OR 2.80, CI 1.49-5.23), melaena (OR 2.59, CI 1.50-4.45), cirrhosis (OR 3.19, CI 1.26-8.06), and urea trend (admission urea/baseline) (OR 1.37 CI 1.081.73). Admission $\mathrm{Hb}<100 \mathrm{~g} / \mathrm{L}$, absolute urea levels and use of anti-platelets or anticoagulation were not significantly associated with HRE. In combination, these biomarkers were superior at predicting HRE compared to pre-existing scoring systems (AUROC 0.765, p=0.000) (Figure 1).

Conclusions A novel scoring system incorporating haematemesis, melaena, presence of cirrhosis, and urea trend may demonstrate a superior ability to predict high-risk features during endoscopy which in-turn predicts poor prognosis in AUGIB. Our data suggests that urea trend may provide additional value compared to absolute urea levels. Interestingly both admission haemoglobin $<100 \mathrm{~g} / \mathrm{L}$ and use of anti-platelets or anti-coagulation did not significantly increase the risk of highrisk features on endoscopy.

\section{REFERENCE}

1. Siau K, Hearnshaw S, Stanley AJ, Estcourt L, Rasheed A, Walden A, et al. British Society of Gastroenterology (BSG)-led multisociety consensus care bundle for the early clinical management of acute upper gastrointestinal bleeding. Frontline Gastroenterology 2020;11(4):311-23.

\section{PTU-8 ENDOSCOPICALLY 'MISSED' UPPER GASTROINTESTINAL CANCERS IN A SINGLE NHS CENTRE OVER 5 YEARS, 2015-19}

Dominic Crocombe*, Robert Kennedy, Prashant Kant. York Teaching Hospitals Nhs Foundation Trust, York, UK

\subsection{6/gutjnl-2021-BSG.81}

Introduction Missed upper gastrointestinal cancer (UGIC) is defined in cases that had not been diagnosed at a previous oesophago-gastroduodenoscopy (OGD) performed within 3 years before the diagnosis. We reviewed the rate of missed UGIC at York Teaching Hospitals NHS Foundation Trust between 201519 to evaluate the service and inform strategies for improvement.

Methods Electronic patient records of all cases referred to the UGIC service between 2015-19 were interrogated for: patient demographics; diagnosis, staging and histology; OGD in 3 years preceding diagnosis that did not lead to diagnosis of UGIC; dates, indications, findings, and grade of endoscopist at diagnostic and prior OGD. Extra-luminal UGICs and those picked up on routine Barrett's screening (unless interval symptoms led to expedited OGD) were excluded.

Results 537 patients were diagnosed with UGIC between 2015-19, with median age 72 years at diagnosis. Of these, 37 (7\%) met the definition of a missed UGIC, with a median age of 73 years at diagnosis. Of these, 16 (43\%) were oesophageal, 5 (14\%) gastro-oesophageal junction, 13 (35\%) gastric, and $3(8 \%)$ duodenal. The mean time between previous OGD and diagnosis in missed UGIC was 16.8 months.

11 of the $16(69 \%)$ missed oesophageal cancers were adenocarcinoma, of which only 2 were in patients with known Barrett's. Of the 13 missed gastric cancers, 12 (92\%) were adenocarcinoma and 1 neuroendocrine tumour (NET). 8 $(62 \%)$ of the missed gastric cancers were in the distal stomach, 3 (23\%) mid-stomach, and $2(15 \%)$ proximal stomach. Of the 3 duodenal cancers, 2 were adenocarcinoma and 1 was a NET.

In 25 of $37(68 \%)$ cases, the endoscopist of the OGD at which the cancer was missed was a consultant, 4 (11\%) GP endoscopist, 3 (8\%) nurse endoscopist, 3 specialist trainee alone, $1(3 \%)$ specialist trainee supervised by consultant.

Conclusions The overall rate of missed UGICs was 7\%, which is comparable to rates at other centres around the world; one meta-analysis quotes a range of 5.7 to $13.1 \%{ }^{1}$ Missed oesophageal cancers were not strongly associated with pre-existing Barrett's, which is reassuring in the context of current screening rates. $62 \%$ of missed gastric cancers were in the distal stomach, which suggests it might be prudent to focus on this anatomical region to improve detection of subtle lesions. Finally, there was no clear pattern regarding role (for example, doctor or nurse) or grade of endoscopist and missed UGI cancer rates.

\section{REFERENCE}

1. Pimenta-Melo et al. Missing rate for gastric cancer during upper gastrointestinal endoscopy: a systematic review and meta-analysis. European Journal of Gastroenterology \& Hepatology 2016;28:1041-49. 